\title{
The Gossypium longicalyx genome as a resource for cotton breeding and evolution
} Corrinne E. Grover ${ }^{1}$, Mengqiao Pan $^{2}$, Daojun Yuan ${ }^{3}$, Mark A. Arick $\mathrm{II}^{4}$, Guanjing $\mathrm{Hu}^{1}$, Logan Brase $^{5}$, David M. Stelly ${ }^{6}$, Zefu Lu ${ }^{7}$, Robert J. Schmitz ${ }^{7}$, Daniel G. Peterson ${ }^{4}$, Jonathan F. Wendel $^{1}$, and Joshua A. Udall ${ }^{8 *}$

${ }^{1}$ Ecology, Evolution, and Organismal Biology Dept., Iowa State University, Ames, IA, 50010

${ }^{2}$ State Key Laboratory of Crop Genetics and Germplasm Enhancement, Cotton Hybrid R \& D Engineering Center, Nanjing Agricultural University, Nanjing, 210095, China

${ }^{3}$ College of Plant Science and Technology, Huazhong Agricultural University, Wuhan, Hubei, 430070, China

${ }^{4}$ Institute for Genomics, Biocomputing \& Biotechnology, Mississippi State University, United States

${ }^{5}$ Division of Biology and Biomedical Sciences, Washington University in St. Louis, St. Louis, MO 63110

${ }^{6}$ Department of Soil and Crop Sciences, Texas A\&M University, College Station, 77843, USA

${ }^{7}$ Department of Genetics, University of Georgia, Athens, GA 30602

${ }^{8}$ USDA/Agricultural Research Service, Crop Germplasm Research Unit, College Station, TX 77845

ORCID (email):

CEG: 0000-0003-3878-5459 (corrinne@iastate.edu)

MP: (mengqiaopan@live.com)

DY: 0000-0001-6007-5571(robert@mail.hzau.edu.cn)

MAA: 0000-0002-7207-3052 (maa146@IGBB.MsState.Edu)

GH: 0000-0001-8552-7394 (hugj2006@iastate.edu)

LB: 0000-0002-7175-3208 (braselogan@gmail.com)

DS: 0000-0002-3468-4119 (stelly@tamu.edu)

ZL: (zefulu@uga.edu)

RJS: 0000-0001-7538-6663 (schmitz@uga.edu)

DGP 0000-0002-0274-5968 (peterson@IGBB.MsState.Edu)

JFW 0000-0003-2258-5081 (jfw@iastate.edu)

JAU 0000-0003-0978-4764 (Joshua.Udall@usda.gov)

\section{*corresponding author: Joshua.Udall@usda.gov}

\section{Keywords:}

Gossypium longicalyx, nematode resistance, cotton fiber, genome sequence, PacBio 


\section{Abstract}

Cotton is an important crop that has made significant gains in production over the last century. Emerging pests such as the reniform nematode have threatened cotton production. The rare African diploid species Gossypium longicalyx is a wild species that has been used as an important source of reniform nematode immunity. While mapping and breeding efforts have made some strides in transferring this immunity to the cultivated polyploid species, the complexities of interploidal transfer combined with substantial linkage drag have inhibited progress in this area. Moreover, this species shares its most recent common ancestor with the cultivated A-genome diploid cottons, thereby providing insight into the evolution of long, spinnable fiber. Here we report a newly generated de novo genome assembly of G. longicalyx. This high-quality genome leveraged a combination of PacBio long-read technology, Hi-C chromatin conformation capture, and BioNano optical mapping to achieve a chromosome level assembly. The utility of the G. longicalyx genome for understanding reniform immunity and fiber evolution is discussed.

\section{Introduction}

Cotton (genus Gossypium) is an important crop which provides the largest natural source of fiber. Colloquially, the term cotton refers to one of four domesticated species, primarily the tetraploid G. hirsutum, which is responsible for over $98 \%$ of cotton production worldwide (Kranthi 2018). Gossypium contains over 50 additional wild species related to the domesticated cottons that serve as potential sources of disease and pest resistance. Among these, Gossypium longicalyx J.B. Hutch. \& B.J.S. Lee is the only representative of the diploid "F-genome" (Wendel and Grover 2015) and the only species with immunity to reniform nematode infection (Yik and Birchfield 1984). Discovered only 60 years ago (Hutchinson and B. J. S. Lee 1958), it is both cytogenetically differentiated from members of the other genome groups (Phillips 1966) and morphologically isolated (Fryxell 1971, 1992). Importantly, G. longicalyx is sister to the Agenome cottons (Wendel and Albert 1992; Wendel and Grover 2015; Chen et al. 2016), i.e., G. arboreum and $G$. herbaceum, the only diploids with long, spinnable fiber.

Interest in the genome of G. longicalyx is two-fold. First, broad-scale screening of the cotton germplasm collection indicates that domesticated cotton lacks appreciable natural resistance to reniform nematode (Birchfield et al. 1963; Yik and Birchfield 1984), and while several other species exhibit degrees of resistance, only G. longicalyx exhibits immunity to infection (Yik and Birchfield 1984). This is significant as reniform nematode has emerged as a major source of cotton crop damage, reducing cotton production by over 205 million bales per year (Lawrence et al. 2015) and accounting for $\sim 11 \%$ of the loss attributable to pests (Khanal et al. 2018). Current reniform resistant lines are derived from complex breeding schemes which are required to introgress reniform immunity from the diploid G. longicalyx into polyploid G. hirsutum (Bell and Robinson 2004; Dighe et al. 2009; Khanal et al. 2018); however, undesirable traits have 
81 accompanied this introgression (Nichols et al. 2010) extreme stunting of seedlings and plants exposed to dense nematode populations, prohibiting commercial deployment (Zheng et al. 2016).

84 The genome of $G$. longicalyx is also valuable because it is phylogenetically sister to the only diploid clade with spinnable fiber (Wendel and Albert, 1992; Wendel and Grover, 2015; Chen et al., 2016), the A-genome species, which contributed the maternal ancestor to polyploid cotton. Consequently, there has been interest in this species as the ancestor to spinnable fiber (Hovav et al. 2008; Paterson et al. 2012), although progress has been limited due to lack of genomic resources in G. longicalyx. Comparisons between the G. longicalyx genome and other cotton genomes, including the domesticated diploids (Du et al. 2018), may provide clues into the evolutionary origin of “long” fiber.

Here we describe a high-quality, de novo genome sequence for G. longicalyx, a valuable resource for understanding nematode immunity in cotton and possibly other species. This genome also provides a foundation to understand the evolutionary origin of spinnable fiber in Gossypium.

\section{Methods \& Materials}

\section{Plant material and sequencing methods} Leaf tissue of mature G. longicalyx (F1-1) was collected from a Brigham Young University (BYU) greenhouse. DNA was extracted using CTAB techniques (Kidwell and Osborn 1992), and the amount recovered was measured via Qubit Fluorometer (ThermoFisher, Inc.). The sequencing library was constructed by the BYU DNA Sequencing Center (DNASC) using only fragments $>18 \mathrm{~kb}$, which were size selected on the BluePippen (Sage Science, LLC) and verified in size using a Fragment Analyzer (Advanced Analytical Technologies, Inc). Twenty-six PacBio cells were sequenced from a single library on the Pacific Biosciences Sequel system. Resulting reads were assembled using Canu V1.6 using default parameters (Koren et al. 2017) to create a sequence assembly called Longicalyx_V1.0, composed of 229 large contigs (Figure 1). 


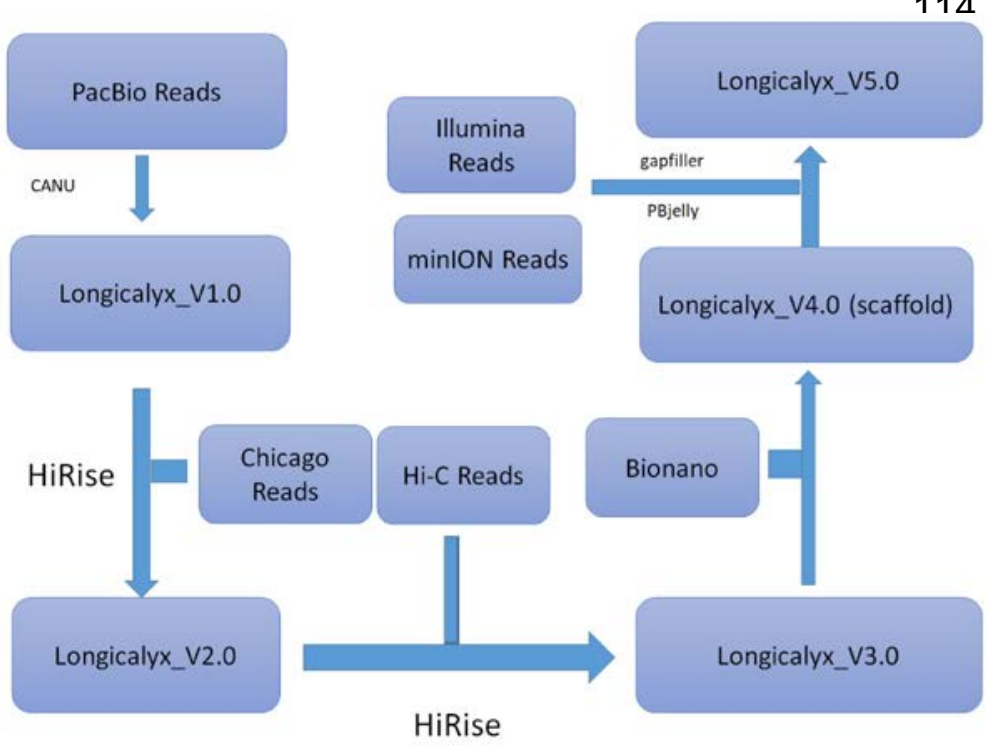

128
Figure 1.

Chicago Highrise reads (Dovetail Genomics) provide DNA-DNA proximity information used to improve the Canu sequence assembly (Longicalyx_V2.0; statistics not calculated), as previously demonstrated for de novo human and alligator genomes (Putnam et al. 2016). Simultaneously, HiC libraries were constructed from $G$. longicalyx leaf tissue at PhaseGenomics LLC. A second

round of HighRise was used to include the HiC data for additional genome scaffolding (Koch 2016; Putnam et al. 2016), reducing the contig number to 135 (Longicalyx_V3.0).

High-molecular weight DNA was extracted from young G. longicalyx leaves and subsequently purified, nicked, labeled, and repaired according to Bionano Plant protocol and standard operating procedures for the Irys platform. BssSI was used in conjunction with the IrysSolve pipeline to assemble an optical map on the BYU Fulton SuperComputing cluster. The resulting optical map was aligned to the assembly named Lonigcalyx_V3.0 using an in silico labeled reference sequence. Bionano maps linked large contigs present in this assembly, producing 17 large scaffolds (Lonigcalyx_V4.0).

Minion sequencing libraries were created and sequenced following the standard protocol from Oxford Nanopore. Scaffolds from Lonigcalyx_V4.0 were polished (Supplemental File 1) with existing Illumina (SRR1174179 and SRR1174182 from the NCBI Short Read Archive) and the newly generated Minion data for G. longicalyx using both PBjelly (English et al. 2012) and GapFiller (Boetzer and Pirovano 2012) to produce the final assembly, Lonigcalyx_V5.0.

\section{Repeat and gene annotation}

Repeats were identified using two methods. The first is a homology-based approach, i.e., a combination of RepeatMasker (Smit et al. 2015) and "One code to find them all” (Bailly-Bechet et al. 2014), whereas the second method (i.e., RepeatExplorer; (Novák et al. 2010) clusters reads based on sequence similarity and automatically annotates the most abundant cluster using RepeatMasker. Each RepeatMasker run used a custom library, which combines Repbase 23.04 repeats (Bao et al. 2015) with cotton-specific repeats. Default parameters were run, except the run was "sensitive” and was set to mask only TEs (no low-complexity). Parameters are available 
https://github.com/Wendellab/longicalyx. "One code to find them all” was used to aggregate multiple hits from the first method (RepeatMasker) into TE models using default parameters. The resulting output was aggregated and summarized in R/3.6.0 (R Core Team 2017) using dplyr/0.8.1 (Wickham et al. 2015). Cluster results were obtained from (Grover et al. 2019) and https://github.com/IGBB/D_Cottons_USDA, and these were parsed in R/3.6.0 (R Core Team 2017). All code is available at https://github.com/Wendellab/longicalyx.

RNA-Seq libraries were generated from G. longicalyx leaf (CL), floral (FF), and stem tissues (FS) to improve genome annotation. RNA-seq libraries were independently constructed by BGI Americas (Davis, CA) using Illumina TruSeq reagents and subsequently sequenced (single-end, $50 \mathrm{bp}$ ). The newly sequenced $G$. longicalyx RNA-seq was combined with existing RNA-seq from G. longicalyx (SRR1174179) as well as two closely related species, i.e., G. herbaceum (developing fibers and seed; PRJNA595350 and SRR959585, respectively) and G. arboreum (5 seed libraries and 1 seedling; SRR617075, SRR617073, SRR617068, SRR617067, SRR959590, and SRR959508). RNA-seq libraries were mapped to the hard-masked G. longicalyx genome using hisat2 [v2.1.0] (Kim et al. 2015). BRAKER2 [v2.1.2] (Hoff et al. 2019) was used in conjunction with GeneMark [v4.36] (Borodovsky and Lomsadze 2011) generated annotations to train Augustus [v3.3.2] (Stanke et al. 2006). Mikado [v1.2.4] (Venturini et al. 2018) was used to produce high quality RNA-seq based gene predictions by combining the RNA-seq assemblies produced by StringTie [v1.3.6] (Pertea et al. 2015) and Cufflinks [v2.2.1] (Ghosh and Chan 2016) with a reference-guided assembly from Trinity [v2.8.5] (Grabherr et al. 2011) and a splice junction analysis from Portcullis [v1.2.2] (Mapleson et al. 2018). The Trinity assembly was formatted using GMAP [v2019-05-12] (Wu and Watanabe 2005). MAKER2 [v2.31.10] (Holt and Yandell 2011; Campbell et al. 2014) was used to integrate gene predictions from (1) BRAKER2 trained Augustus, (2) GeneMark, and (3) Mikado, also using evidence from all Gossypium ESTs available from NCBI (nucleotide database filtered on “txid3633” and “is_est”) and a database composed of all curated proteins in Uniprot Swissprot [v2019_07] (UniProt Consortium 2008) combined with the annotated proteins from the G. hirsutum (https://www.cottongen.org/species/Gossypium_hirsutum/jgi-AD1_genome_v1.1) and G. raimondii (Paterson et al. 2012) genomes. Maker scored each gene model using the annotation edit distance (AED - (Eilbeck et al. 2009; Holt and Yandell 2011; Yandell and Ence 2012) metric based on EST and protein evidence provided. Gene models with an AED greater than 0.47 were removed from further analyses, and the remaining gene models were functionally annotated using InterProScan [v5.35-74.0] (Jones et al. 2014) and BlastP [v2.9.0+] (Camacho et al. 2009) searches against the Uniprot SwissProt database. Orthologs between the G. longicalyx annotations and the existing annotations for G. arboreum (Du et al. 2018), G. raimondii (Paterson et al. 2012), G. hirsutum (Hu et al. 2019), and G. barbadense (Hu et al. 2019) were predicted by OrthoFinder using default settings (Emms and Kelly 2015, 2019). All genomes are hosted through CottonGen (https://www.cottongen.org; (Yu et al. 2014)) and running parameters are available from https://github.com/Wendellab/longicalyx. 


\section{ATAC-seq and data analysis}

196

197

198

199

200

201

202

203

204

205

206

207

208

209

210

211

212

213

214

215

216

217

218

219

220

221

222

223

224

225

226

227

228

229

230

231

232

233

ATAC-seq was performed as described previously (Lu et al. 2017). For each replicate, approximately $200 \mathrm{mg}$ freshly collected leaves or flash frozen leaves were immediately chopped with a razor blade in $\sim 1 \mathrm{ml}$ of pre-chilled lysis buffer $(15 \mathrm{mM}$ Tris- $\mathrm{HCl} \mathrm{pH}$ 7.5, $20 \mathrm{mM} \mathrm{NaCl}$, $80 \mathrm{mM} \mathrm{KCl,} 0.5 \mathrm{mM}$ spermine, $5 \mathrm{mM}$ 2-mercaptoethanol, 0.2\% Triton X-100). The chopped slurry was filtered twice through miracloth and once through a $40 \mu \mathrm{m}$ filter. The crude nuclei were stained with DAPI and loaded into a flow cytometer (Beckman Coulter MoFlo XDP). Nuclei were purified by flow sorting and washed in accordance with Lu et al (Lu et al. 2017). The sorted nuclei were incubated with $2 \mu \mathrm{T}$ Tn 5 transposomes in $40 \mu$ of tagmentation buffer (10 $\mathrm{mM}$ TAPS-NaOH ph 8.0, $5 \mathrm{mM} \mathrm{MgCl}_{2}$ ) at $37^{\circ} \mathrm{C}$ for 30 minutes without rotation. The integration products were purified using a Qiagen MinElute PCR Purification Kit or NEB Monarch ${ }^{\mathrm{TM}}$ DNA Cleanup Kit and then amplified using Phusion DNA polymerase for 10-13 cycles. PCR cycles were determined as described previously (Buenrostro et al. 2013). Amplified libraries were purified with AMPure beads to remove primers. ATAC-seq libraries were sequenced in pairedend 35 bp at the University of Georgia Genomics \& Bioinformatics Core using an Illumina NextSeq 500 instrument.

Reads were adapter and quality trimmed, and then filtered using “Trim Galore” [v0.4.5] (Krueger 2015). Clean reads were subsequently aligned to the Lonigcalyx_V5.0 assembly using Bowtie2 [v2.3.4] (Langmead and Salzberg 2012) with the parameters “--no-mixed --nodiscordant --no-unal --dovetail”. Duplicate reads were removed using Picard [v2.17.0] with default parameters (http://broadinstitute.github.io/picard/). Only uniquely mapped read pairs with a quality score of at least 20 were kept for peak calling. Phantompeakqualtools [v1.14] (Landt et al. 2012) was used to calculate the strand cross-correlation, and deepTools [v2.5.2] (Ramírez et al. 2016) was used to calculate correlation between replicates. The peak calling tool from HOMER [v4.10] (Heinz et al. 2010), i.e., findpeaks, was run in "region” mode and with the minimal distance between peaks set to 150 bp. MACS2 [v2.1.1] (Zhang et al. 2008) callpeak, a second peak-calling algorithm, was run with the parameter "-f BAMPE” to analyze only properly paired alignments, and putative peaks were filtered using default settings and false discovery rate (FDR) $<0.05$. Due to the high level of mapping reproducibility (Pearson's correlation $r=0.99$ and Spearman correlation $r=0.77$ by deepTools), peaks were combined and merged between replicates for each tool using BEDTools [v2.27.1] (Quinlan 2014). BEDTools was also used to intersect HOMER peaks and MACS2 peaks to only retain peak regions identified by both tools as accessible chromatin regions (ACRs) for subsequent analyses.

ACRs were annotated in relation to the nearest annotated genes in the R environment [v3.5.0] as genic (gACRs; overlapping a gene), proximal (pACRs; within $2 \mathrm{~Kb}$ of a gene) or distal (dACRs; $>2 \mathrm{~Kb}$ from a gene). Using R package ChIPseeker [v1.18.0] (Yu et al. 2015), the distribution of ACRs was calculated around transcription start sites (TSS) and transcription termination sites 
(TTS), and peak distribution was visualized with aggregated profiles and heatmaps. To compare was used to generate the distal (by excluding genic and $2 \mathrm{~Kb}$ flanking regions) and genic/proximal control regions (by including genic and $2 \mathrm{~Kb}$ flanking regions), and the nuc command was used to calculate GC content for each ACR and permuted control regions. consistently cosegrates with resistance and that is flanked by the SNP markers Gl_168758 and Gl_072641, which are all located in the region of G. longicalyx chromosome 11 referred to as

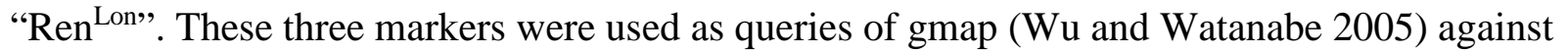
the assembled genome to identify the genomic regions associated with each. The coordinates identified by gmap were placed in a bed file; this file was used in conjunction with the $G$. longicalyx annotation and BEDtools intersect (Quinlan 2014) to identify predicted G. longicalyx genes contained within Ren ${ }^{\text {Lon }}$. Samtools faidx (Li et al. 2009) was used to extract the 52 identified genes from the annotation file, which were functionally annotated using blast2go (blast2go basics; biobam) and including blastx (Altschul et al. 1990), gene ontology (The Gene Ontology Consortium 2019), and InterPro (Jones et al. 2014). Orthogroups containing each of the 52 Ren $^{\text {Lon }}$ genes were identified from the Orthofinder results (see above).

\section{Comparison between $G$. arboreum and G. longicalyx for fiber evolution}

Whole-genome alignments were generated between $G$. longicalyx and either G. arboreum, $G$. raimondii, G. turneri, G. hirsutum (A-chromosomes), and G. barbadense (A-chromosomes) using Mummer (Marçais et al. 2018) and visualized using dotPlotly (https://github.com/tpoorten/dotPlotly) in R (version 3.6.0) (R Development Core Team and Others 2011). Divergence between G. longicalyx and G. arboreum or G. raimondii was calculated using orthogroups that contain a single $G$. longicalyx gene with a single G. arboreum and/or single G. raimondii gene. Pairwise alignments between G. longicalyx and G. arboreum or G. raimondii were generated using the linsi from MAFFT (Katoh and Standley 2013). Pairwise distances between G. longicalyx and G. arboreum and/or G. raimondii were calculated in R (version 3.6.0) using phangorn (Schliep 2011) and visualized using ggplot2 (Wickham 2016). To identify genes unique to species with spinnable fiber (i.e., G. arboreum and the polyploid species), we extracted any G. arboreum gene contained within orthogroups composed solely of G. arboreum or polyploid A-genome gene annotations, and subjected these to blast2go (as above). Syntenic conservation of genes contained within the $\operatorname{Ren}^{\text {Lon }}$ region, as compared to $G$. arboreum, was evaluated using GEvo as implemented in SynMap via COGE (Lyons and 


\section{Data availability}

The assembled genome sequence of G. longicalyx is available at NCBI SUB6483233 and NCBI PRJNA420071 for PacBio and Minion, and PRJNA420070 for RNA-Seq. Supplemental files are available from figshare.

\section{Results and Discussion}

282

\section{Genome assembly and annotation}

We report a de novo genome sequence for G. longicalyx. This genome was first assembled from $\sim 144$ x coverage (raw) of PacBio reads, which alone produced an assembly consisting of 229 contigs with an N50 of 28.8MB (Table 1). The contigs were scaffolded using a combination of Chicago Highrise, Hi-C, and BioNano to produce a chromosome level assembly consisting of 17 contigs with an average length of $70.4 \mathrm{Mb}$ (containing only $8.4 \mathrm{~kb}$ of gap sequence). Thirteen of the chromosomes were assembled into single contigs. Exact placement of the three unscaffolded contigs ( $100 \mathrm{~kb}$ ) was not determined, but these remaining sequences were included in NCBI with the assembled chromosomes. The final genome assembly size was 1190.7 MB, representing over $90 \%$ of the estimated genome size (Hendrix and Stewart 2005).

Table 1. Statistics for assembly versions

\begin{tabular}{lrrrr}
\multicolumn{7}{c}{$\begin{array}{l}\text { G. longicalyx assemblies* } \\
\text { Method }\end{array}$} & $\begin{array}{l}\text { Longicalyx_V1.0 } \\
\text { PacBio/Canu }\end{array}$ & $\begin{array}{c}\text { Longicalyx_V3.0 } \\
\text { +Chicago HighRise+HiC }\end{array}$ & $\begin{array}{l}\text { Longicalyx_V4.0 } \\
\text { +BioNano }\end{array}$ & $\begin{array}{c}\text { Longicalyx_V5.0 } \\
\text { +Illumina+Minion }\end{array}$ \\
Coverage & 79.45 & 135 & 17 & 17 \\
Total Contig Number & 229 & $1196.19 \mathrm{Mb}$ & $1190.66 \mathrm{Mb}$ & $1190.67 \mathrm{Mb}$ \\
Assembly Length** & $1196.17 \mathrm{Mb}$ & $8.86 \mathrm{Mb}$ & $70.04 \mathrm{Mb}$ & $70.04 \mathrm{Mb}$ \\
Average Contig Length & $5.22 \mathrm{Mb}$ & 18200 & 18000 & 8488 \\
Total Length of Ns & 0 & $95.88 \mathrm{Mb}$ & $95.88 \mathrm{Mb}$ & $95.88 \mathrm{Mb}$ \\
N50 value is & $28.88 \mathrm{Mb}$ & $76.48 \mathrm{Mb}$ & $76.48 \mathrm{Mb}$ & $76.29 \mathrm{Mb}$ \\
N90 value is & $7.58 \mathrm{Mb}$ & &
\end{tabular}

* Statistics for Longicalyx_V2.0 not calculated

** Genome size for G. longicalyx is 1311 (Hendrix and Stewart, 2005)

BUSCO analysis of the completed genome (Waterhouse et al. 2017) recovered 95.8\% complete BUSCOs (from the total of 2121 BUSCO groups searched; Table 2). Most BUSCOs (86.5\%) were both complete and single copy, with only 9.3\% BUSCOs complete and duplicated. Less than $5 \%$ of BUSCOs were either fragmented (1.4\%) or missing (2.8\%), indicating a general completeness of the genome. Genome contiguity was independently verified using the LTR Assembly Index (LAI) (Ou et al. 2018), which is a reference-free method to assess genome contiguity by evaluating the completeness of LTR-retrotransposon assembly within the genome. 
This method, applied to over 100 genomes in Phytozome, suggests that an LAI between 10 and 20 should be considered "reference-quality"; the G. longicalyx genome reported here received an LAI score of 10.74. Comparison of the G. longicalyx genome to published cotton genomes (Table 2) suggests that the quality of this assembly is similar or superior to other currently available cotton genomes.

Table 2. BUSCO and LAI scores for the G. longicalyx genome compared to existing cotton genomes.

\begin{tabular}{|c|c|c|c|c|c|c|c|}
\hline & \multicolumn{3}{|c|}{ Complete BUSCO } & \multicolumn{2}{|c|}{ Incomplete BUSCO } & \multirow{2}{*}{ LAI score } & \multirow{2}{*}{ Reference } \\
\hline & Total & Single & Duplicated & Fragmented & Missing & & \\
\hline G. longicalyx & $95.80 \%$ & $86.50 \%$ & $9.30 \%$ & $1.40 \%$ & $2.80 \%$ & 10.74 & \\
\hline G. turneri & $95.80 \%$ & $86.00 \%$ & $9.80 \%$ & $1.00 \%$ & $3.20 \%$ & 8.51 & $\begin{array}{l}\text { (Udall et } \\
\text { al. 2019) }\end{array}$ \\
\hline $\begin{array}{l}\text { G. raimondii } \\
\text { (BYU) }\end{array}$ & $92.80 \%$ & $85.10 \%$ & $7.70 \%$ & $2.70 \%$ & $4.50 \%$ & 10.57 & $\begin{array}{l}\text { (Udall et } \\
\text { al. 2019) }\end{array}$ \\
\hline $\begin{array}{l}\text { G. raimondii } \\
(\mathrm{JGI})\end{array}$ & $98.00 \%$ & $87.30 \%$ & $10.70 \%$ & $0.70 \%$ & $1.30 \%$ & 8.51 & $\begin{array}{l}\text { (Paterson et } \\
\text { al. 2012) }\end{array}$ \\
\hline $\begin{array}{l}\text { G. arboreum } \\
\text { (CRI) }\end{array}$ & $94.70 \%$ & $85.20 \%$ & $9.50 \%$ & $1.00 \%$ & $4.30 \%$ & 12.59 & $\begin{array}{l}\text { (Du et al. } \\
\text { 2018) }\end{array}$ \\
\hline $\begin{array}{l}\text { G. barbadense } \\
\text { 3-79 (HAU v2) }\end{array}$ & $96.30 \%$ & $12.20 \%$ & $84.10 \%$ & $0.80 \%$ & $2.90 \%$ & 10.38 & $\begin{array}{l}\text { (Wang et } \\
\text { al. 2019) }\end{array}$ \\
\hline $\begin{array}{c}\text { G. hirsutum } \\
\text { TM1 (HAU v1) }\end{array}$ & $97.70 \%$ & $14.50 \%$ & $83.20 \%$ & $0.50 \%$ & $1.80 \%$ & 10.61 & $\begin{array}{l}\text { (Wang et } \\
\text { al. 2019) }\end{array}$ \\
\hline
\end{tabular}

Genome annotation produced 40,181 transcripts representing 38,378 unique genes. 2012) and G. arboreum (Du et al. 2018) recovered 37,223 and 40,960 genes, respectively. Ortholog analysis between $G$. longicalyx and both diploids suggests a simple 1:1 relationship between a single G. longicalyx gene and a single G. raimondii or G. arboreum gene for 67-68\% of the G. longicalyx genes (25,637 and 26,249 genes, respectively; Table 3). Approximately 3$4 \%$ of the G. longicalyx genome (i.e., 1,153-1,438 genes) are in “one/many” (Table 3) relationships whereby one or more $G$. longicalyx gene model(s) matches one or more $G$. raimondii or G. arboreum gene model(s). The remaining 5,009 genes were not placed in orthogroups with any other cotton genome, slightly higher than the 2,016 - 2,556 unplaced genes in the other diploid species used here. While this could be partly due to genome annotation differences in annotation pipelines, it is also likely due to differences in the amount of RNA-seq available for each genome. 
Table 3. Orthogroups between G. longicalyx and two related diploid species. Numbers of genes are listed and percentages are in parentheses. Relationships listed in the last four lines of the table represent one/many G. longicalyx genes relative to one or many genes from G. arboreum or G. raimondii.

\begin{tabular}{|l|r|r|r|}
\hline & G. longicalyx & G.arboreum & G. raimondii \\
\hline Number of genes & 38,378 & 40,960 & 37,223 \\
\hline Genes in orthogroups & $\begin{array}{r}33,369 \\
(86.9 \%)\end{array}$ & $\begin{array}{r}38,404 \\
(93.8 \%)\end{array}$ & $\begin{array}{r}35,207 \\
(94.6 \%)\end{array}$ \\
\hline Unassigned genes & $5,009(13.1 \%)$ & $2,556(6.2 \%)$ & $2,016(5.4 \%)$ \\
\hline Orthogroups containing species & 26,591 & 29,763 & 29,153 \\
\hline Genes in species-specific orthogroups & $(78.5 \%)$ & $(87.8 \%)$ & $(86.0 \%)$ \\
\hline 1-to-1 relationship & $74(0.2 \%)$ & 0 & $8(0.0 \%)$ \\
\hline 1-to-many relationship & & 26,249 & 25,637 \\
\hline many-to-1 relationship & & $1,207(3.2 \%)$ & $1,153(3.1 \%)$ \\
\hline many-to-many relationship & & $1,438(3.9 \%)$ & $1,172(3.1 \%)$ \\
\hline
\end{tabular}

\section{Repeats}

Transposable element (TE) content was predicted for the genome, both by de novo TE prediction (Bailly-Bechet et al. 2014; Smit et al. 2015) and repeat clustering (Novák et al. 2010). Between 44 - 50\% of the G. longicalyx genome is inferred to be repetitive by RepeatMasker and RepeatExplorer, respectively. While estimates for TE categories (e.g., DNA, Ty3/gypsy, Ty1/copia, etc.) were reasonably consistent between the two methods (Table 4), RepeatExplorer recovered nearly 100 additional megabases of putative repetitive sequences, mostly in the categories of Ty3/gypsy, unspecified LTR elements, and unknown repetitive elements. Interestingly, RepeatMasker recovered a greater amount of sequence attributable to Ty1/copia and DNA elements (Table 4); however, this only accounted for $22 \mathrm{Mb}$ (less than $20 \%$ of the total differences over all categories). The difference between methods with respect to each category and the total TE annotation is relatively small and may be attributable to a combination of methods (homology-based TE identification method versus similarity clustering), the underexploration of the cotton TE population, and sensitivity differences in each method with respect to TE age/abundance.

Table 4. Comparison between repeat quantification methods for the G. longicalyx genome. Amounts are given in megabases $(\mathrm{Mb})$.

$\begin{array}{lrr} & \text { RepeatExplorer } & \text { RepeatMasker/OneCode } \\ \text { LTR/Gypsy (Ty3) } & 557 & 513 \\ \text { LTR/Copia (Ty1) } & 39 & 48 \\ \text { LTR, unspecified } & 44 & 0 \\ \text { DNA (all element types) } & 2.3 & 15 \\ \text { unknown } & 18 & 0 \\ \text { Total repetitive clustered } & 660 & 575\end{array}$


Because the RepeatExplorer pipeline allows simultaneous analysis of multiple samples (i.e., coclustering), we used that repeat profile for both description and comparison to the closely related sister species, G. herbaceum and G. arboreum (from subgenus Gossypium). Relative to other cotton species, G. longicalyx has an intermediate amount of TEs, as expected from its intermediate genome size (1311 Mb; genome size range for Gossypium diploids = 841 - 2778 $\mathrm{Mb})$. Approximately half of the genome $(660 \mathrm{Mb})$ is composed of repetitive sequences, somewhat less than the closely related sister (A-genome) clade, which are slightly bigger in total size and have slightly more repetitive sequence ( $60 \%$ repetitive; Table 5 ). Over $80 \%$ of the $G$. longicalyx repetitive fraction is composed of Ty3/gypsy elements, a similar proportion to the proportion of Ty3/gypsy in subgenus Gossypium genomes. Most other element categories were roughly similar in total amount and proportion between $G$. longicalyx and the two species from subgenus Gossypium (Figure 2).

Table 5. Transposable element content in G. longicalyx versus the sister clade (section Gossypium)

\section{Subgenus Longiloba}

G. longicalyx

Genome Size

LTR/Gypsy (Ty3)

LTR/Copia (Ty1)

LTR, unspecified

DNA (all element types)

unknown

Total repetitive clustered

$\%$ genome is repetitive

$\%$ genome is gypsy

$\%$ repetitive is gypsy
1311

557

39

44

2.3

18

660

$50 \%$

$42 \%$

$84 \%$
Subgenus Gossypium

G. herbaceum

1667

876

43

62

2.7

27

1011

$61 \%$

$53 \%$

$87 \%$
$62 \%$

$55 \%$

1711

943

41

57

2.4

1067

$88 \%$ 


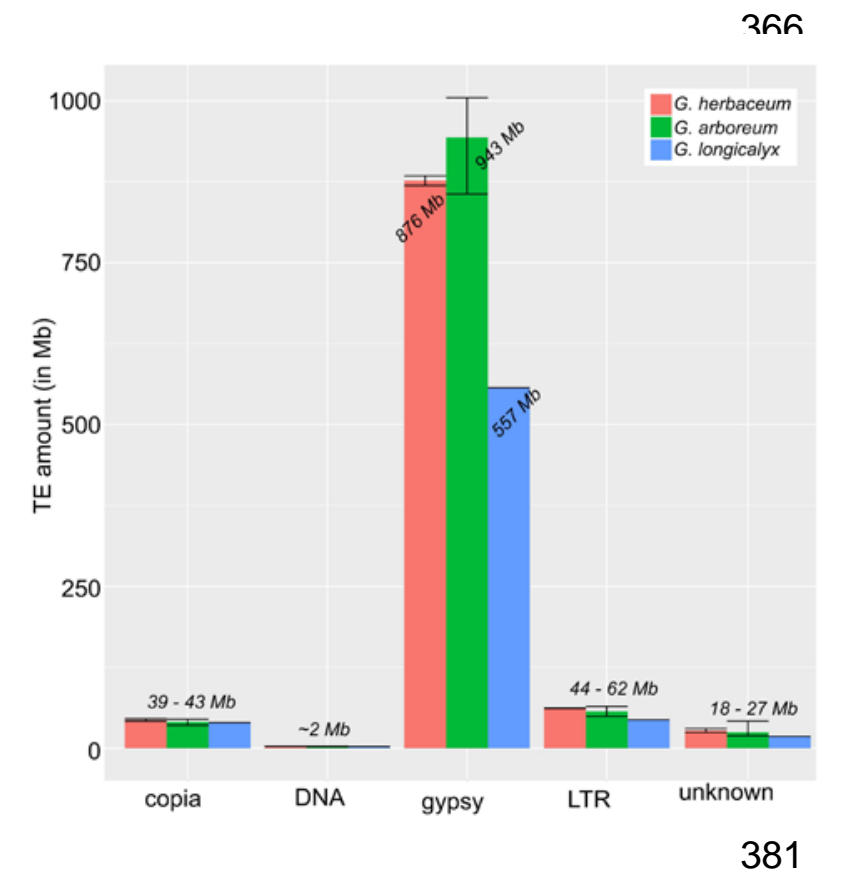

Figure 2. Repetitive content in G. longicalyx relative to the related diploid species $G$. herbaceum and G. arboreum.

Chromatin accessibility in G. longicalyx

We performed ATAC-seq to map accessible chromatin regions (ACRs) in leaves. Two replicated ATAC-seq libraries were sequenced to $\sim 25.7$ and $\sim 45.0$ million reads per sample. The strand cross-correlation statistics supported the high quality of the ATAC-seq data, and the correlation of mapping read coverages (Pearson $r=0.99$ and Spearman $r=0.77$ ) suggested a high level of reproducibility between replicates (Supplemental Table 1). A total of 28,030 ACRs (6.4 Mb) were identified ranging mostly from 130 bp to 400 bp in length, which corresponds to $0.5 \%$ of the assembled genome size (Supplemental Table 2). The enrichment of ACRs around gene transcription start sites (Supplemental Figure 1) suggested that these regions were functionally important and likely enriched with cis-regulatory elements. Based on proximity to their nearest annotated genes, these ACRs were categorized as genic (gACRs; overlapping a gene), proximal (pACRs; within $2 \mathrm{~Kb}$ of a gene) or distal (dACRs; $>2 \mathrm{~Kb}$ from a gene). The gACRs and pACRs represented $12.2 \%$ and $13.2 \%$ of the total number of ACRs (952 Kb and $854 \mathrm{~Kb}$ in size, respectively), while approximately $75 \%$ (4.6 Mb) were categorized as dACRs, a majority of which were located over $30 \mathrm{~Kb}$ from the nearest gene (Figure 3). This high percentage of dACRs is greater than expected ( $\sim 40 \%$ of $1 \mathrm{~GB}$ genome) given previous ATAC-seq studies in plants ( $\mathrm{Lu}$ et al. 2019; Ricci et al. 2019) and may reflect challenges in annotating rare transcripts. While more thorough, species-specific RNA-seq will improve later annotation versions and refine our understanding of ACR proximity to genes, we do note that our observation of abundant dACRs and potentially long-range cis-regulatory elements is consistent with previous results ( $\mathrm{Lu}$ et al. 2019; Ricci et al. 2019) The dACRs discovered here were the most GC-rich, followed by gACRs and pACRs (52\%, 46\%, and 44\%, respectively), all of whom had GC contents significantly higher than randomly selected control regions with the same length distribution (Figure 3d). Because high GC content is associated with several distinct features that can affect the cis- 
409

a

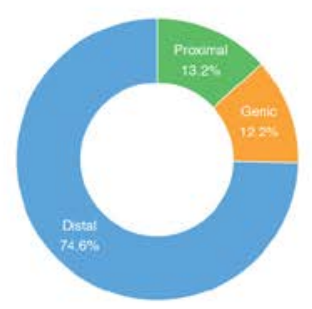

b

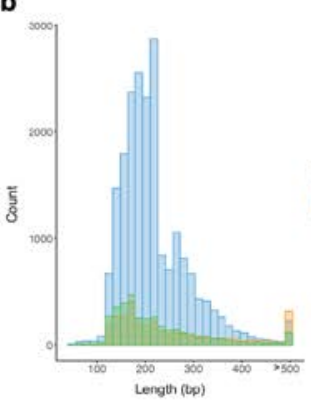

c

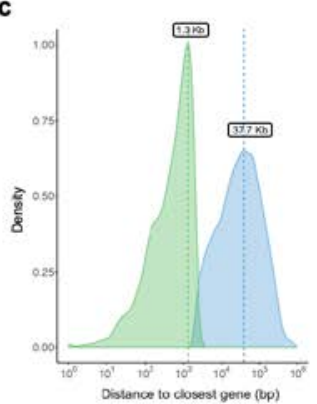

d

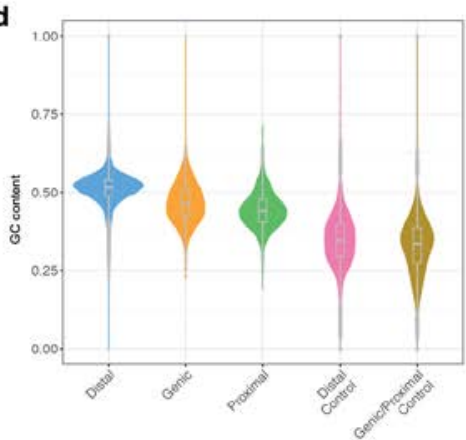

Figure 3. Accessible chromatin regions (ACRs) in the G. longicalyx genome. a. Categorization of ACRs in relation to nearest gene annotations - distal dACRs, proximal pACRs, and genic gACRs. b. Length distribution of ACRs that were identified by both HOMER and MACS2 contained within various genomic regions. c. Distance of gACRs and pACRs to nearest annotated genes. d. Boxplot of GC content in ACRs and control regions.

Genomics of G. longicalyx reniform nematode resistance

Reniform nematode is an important cotton parasite that results in stunted growth, delayed flowering and/or fruiting, and a reduction in both yield quantity and quality (Robinson 2007; Khanal et al. 2018). While domesticated cotton varieties are largely vulnerable to reniform nematode (Robinson et al. 1997), nematode resistance is found in some wild relatives of domesticated cotton, including G. longicalyx, which is nearly immune (Yik and Birchfield 1984). Recent efforts to elucidate the genetic underpinnings of this resistance in G. longicalyx (i.e., Ren $^{\text {Lon }}$ ) identified a marker (BNL1231) that consistently cosegrates with resistance and is flanked by the SNP markers Gl_168758 and Gl_072641 (Dighe et al. 2009; Zheng et al. 2016). Located in chromosome 11, this region contains one or more closely-linked nearly dominant gene(s) (Dighe et al. 2009) that confer hypersensitivity to reniform infection (Khanal et al. 2018), resulting in the "stunting” phenotype; however, the possible effects of co-inherited Rgenes has not been eliminated. Because the introgressed segment recombines at reduced rates in interspecific crosses, it has been difficult to fine-map the gene(s) of interest. Additionally, progress from marker-assisted selection has been lacking, as no recombinants have possessed the desired combination of reniform resistance and "non-stunting” (Zheng et al. 2016). Therefore, more refined knowledge of the position, identity of the resistance gene(s), mode(s) of immunity 


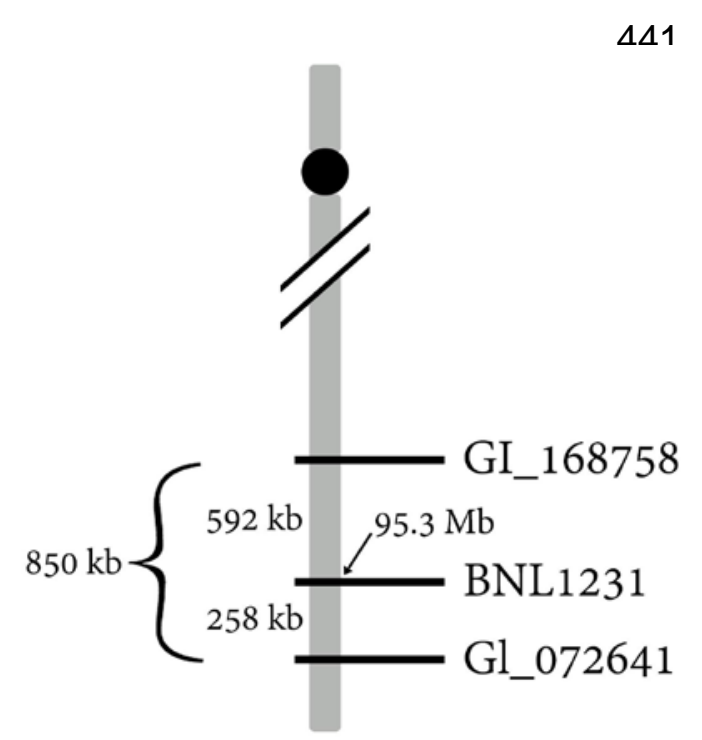

length F11: 99.6 Mb
Figure 4. Diagram of the Ren ${ }^{\text {Lon }}$ region in $G$. longicalyx. Marker BNL1231, which co-segregates with nematode resistance, is located at approximately 95.3 Mb on chromosome F11.

BLAST analysis of the three Ren ${ }^{\text {Lon }}$-associated markers (above) to the assembled G. longicalyx genome identifies an $850 \mathrm{~kb}$ region on chromosome F11 (positions 94747040..95596585; Figure 4) containing 52 predicted genes (Supplemental Table 3). Functional annotation reveals that over half of the genes (29, or $56 \%$ ) are annotated as "TMV resistance protein $\mathrm{N}$-like” or similar. In tobacco, 456 TMV resistance protein $\mathrm{N}$ confers a hypersensitive response to the presence of the tobacco mosaic virus (TMV; (Erickson et al. 1999). Homologs of this gene in different species can confer resistance to myriad other parasites and pathogens, including aphid and nematode resistance in tomato (Rossi et al. 1998); fungal resistance in potato (Hehl et al. 1999) and flax (Ellis et al. 2007); and viral resistance in pepper (Guo et al. 2017). Also included in this region are 6 genes annotated as strictosidine synthase-like (SSL), which may also function in immunity and defense (Sohani et al. 2009). While the six SSL-like genes are tandemly arrayed without disruption, several other genes are intercalated within the array of TMV resistance-like genes, including the 6 SSL-like genes (Supplemental Table 3).

Because there is agronomic interest in transferring nematode resistance from G. longicalyx to other species, we generated orthogroups between G. longicalyx, the two domesticated polyploid species (i.e., G. hirsutum and G. barbadense), and their model diploid progenitors (G. raimondii and G. arboreum; Supplemental Table 4; Supplemental File 2). Interestingly, many of the defense-relevant $G$. longicalyx genes in the Ren ${ }^{\text {Lon }}$ region did not cluster into orthogroups with any other species (15 out of 38; Table 6), including 11 of the 29 TMV resistance-related genes in the Ren $^{\text {Lon }}$ region, and fewer were found in syntenic positions in G. arboreum. Most of the TMV resistance-related genes that cluster between G. longicalyx and other Gossypium species are present in a single, large orthogroup (OG0000022; Table 4), whereas the remaining TMVresistance like genes from $G$. longicalyx are commonly in single gene orthogroups. Since disease resistance $(\mathrm{R})$ proteins operate by detecting specific molecules elicited by the pathogen during infection (Martin et al. 2003), the increased copy number and variability among the $G$. longicalyx TMV-resistance-like genes may suggest specialization among copies. 
Table 6: Orthogroup identity (by Orthofinder) for defense-related genes in the Ren ${ }^{\text {Lon }}$ region and the copy number per species. In G. longicalyx, this number includes genes found outside of the Ren ${ }^{\text {Lon }}$ region. G. hirsutum and G. barbadense copy numbers are split genes found on the A or D chromosomes, or on scaffolds/contigs not placed on a chromosome.

\begin{tabular}{|c|c|c|c|c|c|c|c|}
\hline Description & Orthogroup & $\begin{array}{c}\text { G. longicalyx gene in } \operatorname{Ren}^{\text {Lon }} \\
\text { region }\end{array}$ & $\begin{array}{c}\text { G. } \\
\text { longicalyx }\end{array}$ & $\begin{array}{c}\text { G. } \\
\text { arboreum }\end{array}$ & $\begin{array}{l}\text { G. } \\
\text { raimondii }\end{array}$ & $\begin{array}{c}\text { G. } \\
\text { hirsutum }\end{array}$ & $\begin{array}{c}\text { G. } \\
\text { barbadense }\end{array}$ \\
\hline adenylyl-sulfate kinase 3-like & OG0053444 & Golon.011G359300* & 1 & --- & --- & --- & --- \\
\hline $\begin{array}{l}\text { L-type lectin-domain containing receptor } \\
\text { kinase IV.2-like }\end{array}$ & OG0053450 & Golon.011G361200 & 1 & --- & --- & --- & --- \\
\hline T-complex protein 1 subunit theta-like & OG0053447 & Golon.011G360400 & 1 & --- & --- & --- & --- \\
\hline \multirow{6}{*}{$\begin{array}{l}\text { protein STRICTOSIDINE SYNTHASE- } \\
\text { LIKE 10-like }\end{array}$} & \multirow[t]{5}{*}{ OG0000242 } & Golon.011G363400 & \multirow[t]{5}{*}{6} & \multirow[t]{5}{*}{4} & \multirow[t]{5}{*}{2} & \multirow[t]{5}{*}{$6 \mathrm{~A}$} & \multirow[t]{5}{*}{9 A, 5 scaffold } \\
\hline & & Golon.011G363500 & & & & & \\
\hline & & Golon.011G363600** & & & & & \\
\hline & & Golon.011G363700 & & & & & \\
\hline & & Golon.011G363800 & & & & & \\
\hline & OG0053454 & Golon.011G363300 & 1 & --- & --- & --- & --- \\
\hline \multirow[t]{15}{*}{ TMV resistance protein $\mathrm{N}$-like } & \multirow[t]{15}{*}{ OG0000022 } & Golon.011G360100 & \multirow[t]{15}{*}{25} & \multirow[t]{15}{*}{22} & \multirow[t]{15}{*}{5} & \multirow[t]{15}{*}{$10 \mathrm{~A}, 22 \mathrm{D}$} & \multirow{15}{*}{$\begin{array}{l}12 \text { A, } 21 \mathrm{D}, 1 \\
\text { scaffold }\end{array}$} \\
\hline & & Golon.011G360300 & & & & & \\
\hline & & Golon.011G360500 & & & & & \\
\hline & & Golon.011G360700 & & & & & \\
\hline & & Golon.011G360800 & & & & & \\
\hline & & Golon.011G361000 & & & & & \\
\hline & & Golon.011G361100 & & & & & \\
\hline & & Golon.011G361400 & & & & & \\
\hline & & Golon.011G361900 & & & & & \\
\hline & & Golon.011G362000 & & & & & \\
\hline & & Golon.011G362400 & & & & & \\
\hline & & Golon.011G362700 & & & & & \\
\hline & & Golon.011G362800 & & & & & \\
\hline & & Golon.011G362900* & & & & & \\
\hline & & Golon.011G364000 & & & & & \\
\hline
\end{tabular}


Golon.011G359900

OG0028874* Golon.011G362600

OG0028544 Golon.011G363200

OG0030067 Golon.011G360200

OG0030069 Golon.011G362500

OG0053445 Golon.011G359800

OG0053446 Golon.011G360000

OG0053448 Golon.011G360600

OG0053451 Golon.011G361700

OG0053452 Golon.011G361800

OG0053453 Golon.011G362100

TMV resistance protein N-like isoform X1 OG0053449 Golon.011G360900

OG0028874* Golon.011G362300

TMV resistance protein N-like isoform X2 OG0033549 Golon.011G363900

4

3

1

1

1

1

1

* This gene is syntenically conserved with G. arboreum in the COGE-GEVO analysis.

** This orthogroup is split between two related, but separately named, annotations. 


\section{Comparative genomics and the evolution of spinnable fiber} composed of the A-genome cottons G. arboreum and G. herbaceum. Whereas G. longicalyx fibers are short and tightly adherent to the seed, A-genome fibers are longer and suitable for spinning. Accordingly, there has been interest in the changes in the A-genome lineage that have led to spinnable fiber (Hovav et al. 2008; Paterson et al. 2012). Progress here has been limited by the available resources for G. longicalyx, relying on introgressive breeding (Nacoulima et al. 2012), microarray expression characterization (Hovav et al. 2008), and SNP-based surveys (Paterson et al. 2012) of G. longicalyx genes relative to G. herbaceum. As genomic resources and surveys for selection are becoming broadly available for the A-genome cottons, our

493

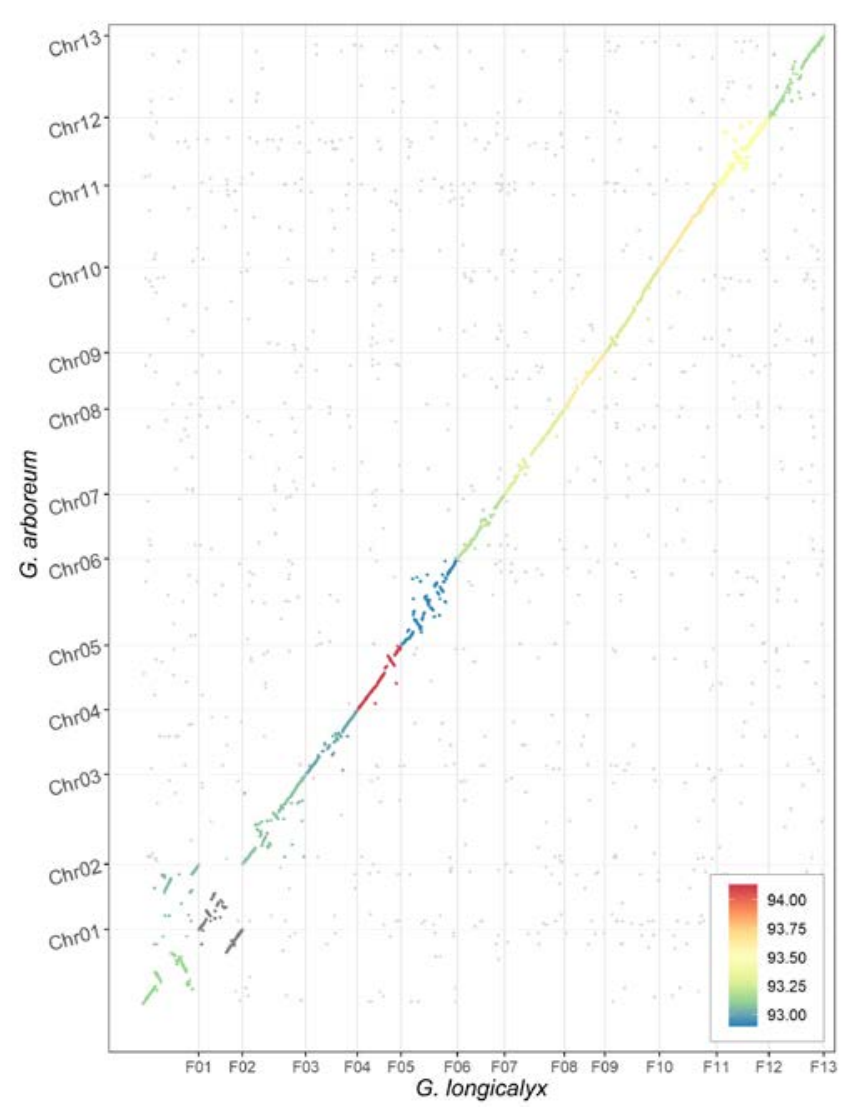

514 understanding of the evolution of spinnable fiber becomes more tangible by the inclusion of G. longicalyx.

Figure 5: Synteny between G. longicalyx and domesticated G. arboreum. Mean percent identity is illustrated by the color (93-94\% identity from blue to red), including intergenic regions.

Whole-genome alignment between $G$. longicalyx and the closely related $G$. arboreum (domesticated for long fiber) shows high levels of synteny and overall sequence identity (Figure 5). In general, these two genomes are largely collinear, save for scattered rearrangements and several involving chromosomes 1 and 2; these latter may represent a combination of chromosomal

515 evolution and/or misassembly in one or both genomes. Notably, comparison of G. longicalyx to 516 other recently published genomes (Supplemental Figures 2-5) suggests that an inversion in the 517 middle of G. longicalyx Chr01 exists relative to representatives of the rest of the genus; however, 518 the other structural rearrangements are restricted to G. arboreum and its derived A subgenome in 519 G. hirsutum and G. barbadense, suggesting that these differences are limited to comparisons 520 between G. longicalyx and A-(sub)genomes. 
522 Genic comparisons between G. longicalyx and G. arboreum suggests a high level of conservation. Orthogroup analysis finds a one-to-one relationship between these two species for over $70 \%$ of genes. Most of these putative orthologs exhibit $<5 \%$ divergence (p-distance) in the coding regions, with over $50 \%$ of all putative orthologs exhibiting less than $1.5 \%$ divergence. Comparatively, the median divergence for putative orthologs between $G$. longicalyx and the more distantly related $G$. raimondii is approximately $2 \%$, with ortholog divergence generally being higher in the $G$. raimondii comparison (Figure 6).

529

530

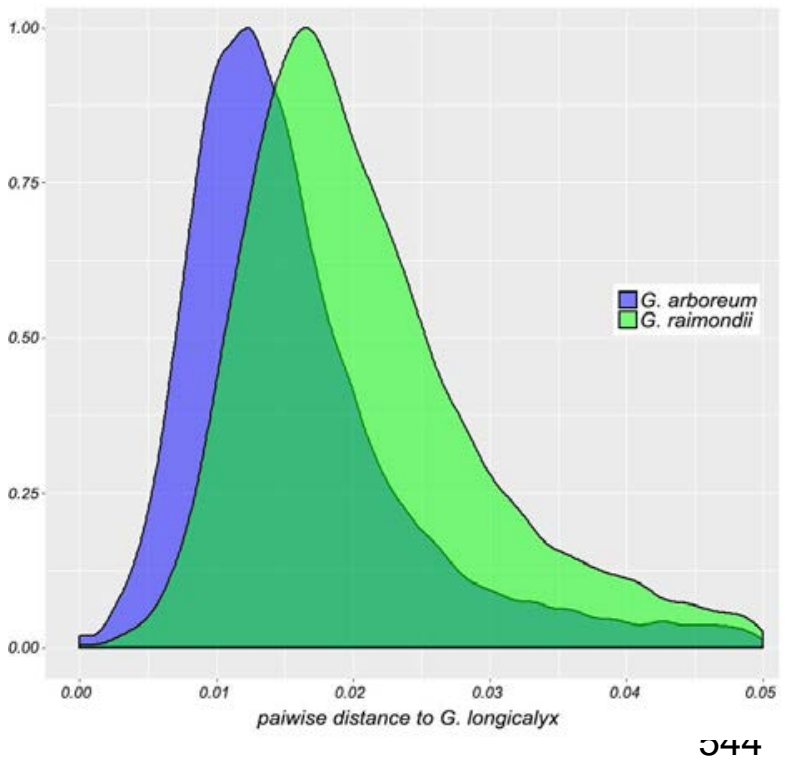

Figure 6: Distribution of pairwise p-distances between coding regions of predicted orthologs (i.e., exons only, start to stop) between $G$. longicalyx and either G. arboreum (blue) or $G$. raimondii (green). Only orthologs with $<5 \%$ divergence are shown, which comprises most orthologs in each comparison.

Because G. longicalyx represents the ancestor to spinnable fiber, orthogroups containing only $G$. arboreum or polyploid A-genome gene annotations may represent genes important in fiber evolution. Accordingly, we extracted 705 G.

545 arboreum genes from orthogroups composed solely of G. arboreum or polyploid (i.e., G.

546 hirsutum or G. barbadense) A-genome gene annotations for BLAST and functional annotation.

547 Of these 705 genes, only 20 represent genes known to influence fiber, i.e., ethylene responsive

548 genes (10), auxin responsive genes (5), and peroxidase-related genes (5 genes; Supplemental

549 Table 4). While other genes on this list may also influence the evolution of spinnable fiber,

550 identifying other candidates will require further study involving comparative coexpression

551 network analysis or explicit functional studies.

\section{Conclusion}

554 While several high-quality genome sequences are available for both wild and domesticated 555 cotton species, each new species provides additional resources to improve both our 556 understanding of evolution and our ability to manipulate traits within various species. In this 557 report, we present the first de novo genome sequence for G. longicalyx, a relative of cultivated 558 cotton. This genome not only represents the ancestor to spinnable fiber, but also contains the 559 agronomically desirable trait of reniform nematode immunity. This resource forms a new 560 foundation for understanding the source and mode of action that provides G. longicalyx with this 
561 valuable trait, and will facilitate efforts in understanding and exploiting it in modern crop

562 species.

\section{Acknowledgements}

We thank Emma Miller and Evan Long for technical assistance. We thank the National Science Foundation Plant Genome Research Program (Grant \#1339412) and Cotton Inc. for their financial support. This research was funded, in part, through USDA ARS Agreements 58-60666-046 and 58-6066-6-059. Support for R.J.S and Z.L. was provided by NSF IOS-1856627 and the Pew Charitable Trusts. We thank BYU Fulton SuperComputer lab for their resources and generous support. We also thank ResearchIT for computational support at Iowa State University.

We thank Rise Services for office accommodations in Orem, UT.

\section{References}

Altschul, S. F., W. Gish, W. Miller, E. W. Myers, and D. J. Lipman, 1990 Basic local alignment search tool. J. Mol. Biol. 215: 403-410.

Bailly-Bechet, M., A. Haudry, and E. Lerat, 2014 "One code to find them all”: a perl tool to conveniently parse RepeatMasker output files. Mob. DNA 5: 13.

Bao, W., K. K. Kojima, and O. Kohany, 2015 Repbase Update, a database of repetitive elements in eukaryotic genomes. Mob. DNA 6: 11.

Bell, A., and A. F. Robinson, 2004 Development and characteristics of triple species hybrids used to transfer reniform nematode resistance from Gossypium longicalyx to Gossypium hirsutum, pp. 422-426 in Proceedings of the Beltwide Cotton Conferences, naldc.nal.usda.gov.

Birchfield, W., L. R. Brister, and Others, 1963 Susceptibility of cotton and relatives to reniform nematode in Louisiana. Plant Disease Reporter 47: 990-992.

Boetzer, M., and W. Pirovano, 2012 Toward almost closed genomes with GapFiller. Genome Biol. 13: R56.

Borodovsky, M., and A. Lomsadze, 2011 Eukaryotic gene prediction using GeneMark.hmm-E and GeneMark-ES. Curr. Protoc. Bioinformatics Chapter 4: Unit 4.6.1-10.

Buenrostro, J. D., P. G. Giresi, L. C. Zaba, H. Y. Chang, and W. J. Greenleaf, 2013 Transposition of native chromatin for fast and sensitive epigenomic profiling of open chromatin, DNA-binding proteins and nucleosome position. Nat. Methods 10: 1213-1218.

Camacho, C., G. Coulouris, V. Avagyan, N. Ma, J. Papadopoulos et al., 2009 BLAST+: architecture and applications. BMC Bioinformatics 10: 421.

Campbell, M. S., C. Holt, B. Moore, and M. Yandell, 2014 Genome Annotation and Curation Using MAKER and MAKER-P. Curr. Protoc. Bioinformatics 48: 4.11.1-39.

Chen, Z., K. Feng, C. E. Grover, P. Li, F. Liu et al., 2016 Chloroplast DNA Structural Variation, Phylogeny, and Age of Divergence among Diploid Cotton Species. PLoS One 11: e0157183.

Dighe, N. D., A. F. Robinson, A. A. Bell, M. A. Menz, R. G. Cantrell et al., 2009 Linkage 
601

602

603

604

605

606

607

608

609

610

611

612

613

614

615

616

617

618

619

620

621

622

623

624

625

626

627

628

629

630

631

632

633

634

635

636

637

638

639

640

Mapping of Resistance to Reniform Nematode in Cotton following Introgression from Gossypium longicalyx (Hutch. \& Lee). Crop Sci. 49: 1151-1164.

Du, X., G. Huang, S. He, Z. Yang, G. Sun et al., 2018 Resequencing of 243 diploid cotton accessions based on an updated A genome identifies the genetic basis of key agronomic traits. Nat. Genet. 50: 796-802.

Eilbeck, K., B. Moore, C. Holt, and M. Yandell, 2009 Quantitative measures for the management and comparison of annotated genomes. BMC Bioinformatics 10: 67.

Ellis, J. G., P. N. Dodds, and G. J. Lawrence, 2007 Flax rust resistance gene specificity is based on direct resistance-avirulence protein interactions. Annu. Rev. Phytopathol. 45: 289-306.

Emms, D. M., and S. Kelly, 2019 OrthoFinder: phylogenetic orthology inference for comparative genomics. bioRxiv 466201.

Emms, D. M., and S. Kelly, 2015 OrthoFinder: solving fundamental biases in whole genome comparisons dramatically improves orthogroup inference accuracy. Genome Biol. 16: 157.

English, A. C., S. Richards, Y. Han, M. Wang, V. Vee et al., 2012 Mind the gap: upgrading genomes with Pacific Biosciences RS long-read sequencing technology. PLoS One 7: e47768.

Erickson, F. L., S. Holzberg, A. Calderon-Urrea, V. Handley, M. Axtell et al., 1999 The helicase domain of the TMV replicase proteins induces the N-mediated defence response in tobacco. Plant J. 18: 67-75.

Fryxell, P. A., 1992 A revised taxonomic interpretation of Gossypium L (Malvaceae). Rheeda 2: 108-165.

Fryxell, P. A., 1971 PHENETIC ANALYSIS AND THE PHYLOGENY OF THE DIPLOID SPECIES OF GOSSYPIUM L. (MALVACEAE). Evolution 25: 554-562.

Ghosh, S., and C.-K. K. Chan, 2016 Analysis of RNA-Seq Data Using TopHat and Cufflinks. Methods Mol. Biol. 1374: 339-361.

Grabherr, M. G., B. J. Haas, M. Yassour, J. Z. Levin, D. A. Thompson et al., 2011 Full-length transcriptome assembly from RNA-Seq data without a reference genome. Nat. Biotechnol. 29: 644-652.

Grover, C. E., M. A. Arick 2nd, A. Thrash, J. L. Conover, W. S. Sanders et al., 2019 Insights into the Evolution of the New World Diploid Cottons (Gossypium, Subgenus Houzingenia) Based on Genome Sequencing. Genome Biol. Evol. 11: 53-71.

Guo, G., S. Wang, J. Liu, B. Pan, W. Diao et al., 2017 Rapid identification of QTLs underlying resistance to Cucumber mosaic virus in pepper (Capsicum frutescens). Theor. Appl. Genet. 130: 41-52.

Haug-Baltzell, A., S. A. Stephens, S. Davey, C. E. Scheidegger, and E. Lyons, 2017 SynMap2 and SynMap3D: web-based whole-genome synteny browsers. Bioinformatics 33: 21972198.

Hehl, R., E. Faurie, J. Hesselbach, F. Salamini, S. Whitham et al., 1999 TMV resistance gene N homologues are linked to Synchytrium endobioticum resistance in potato. Theor. Appl. Genet. 98: 379-386. 
641

642

643

644

645

646

647

648

649

650

651

652

653

654

655

656

657

658

659

660

661

662

663

664

665

666

667

668

669

670

671

672

673

674

675

676

677

678

679

680

Heinz, S., C. Benner, N. Spann, E. Bertolino, Y. C. Lin et al., 2010 Simple Combinations of Lineage-Determining Transcription Factors Prime cis-Regulatory Elements Required for Macrophage and B Cell Identities. Molecular Cell 38: 576-589.

Hendrix, B., and J. M. Stewart, 2005 Estimation of the nuclear DNA content of gossypium species. Ann. Bot. 95: 789-797.

Hoff, K. J., A. Lomsadze, M. Borodovsky, and M. Stanke, 2019 Whole-Genome Annotation with BRAKER. Methods Mol. Biol. 1962: 65-95.

Holt, C., and M. Yandell, 2011 MAKER2: an annotation pipeline and genome-database management tool for second-generation genome projects. BMC Bioinformatics 12: 491.

Hovav, R., J. A. Udall, B. Chaudhary, E. Hovav, L. Flagel et al., 2008 The evolution of spinnable cotton fiber entailed prolonged development and a novel metabolism. PLoS Genet. 4: e25.

Hu, Y., J. Chen, L. Fang, Z. Zhang, W. Ma et al., 2019 Gossypium barbadense and Gossypium hirsutum genomes provide insights into the origin and evolution of allotetraploid cotton. Nat. Genet. 51: 739-748.

Hutchinson, J. B., and B. J. S. Lee, 1958 Notes from the East African Herbarium: IX: A New Species of Gossypium from Central Tanganyika. Kew Bull. 13: 221-223.

Jones, P., D. Binns, H.-Y. Chang, M. Fraser, W. Li et al., 2014 InterProScan 5: genome-scale protein function classification. Bioinformatics 30: 1236-1240.

Katoh, K., and D. M. Standley, 2013 MAFFT multiple sequence alignment software version 7: improvements in performance and usability. Mol. Biol. Evol. 30: 772-780.

Khanal, C., E. C. McGawley, C. Overstreet, and S. R. Stetina, 2018 The Elusive Search for Reniform Nematode Resistance in Cotton. Phytopathology 108: 532-541.

Kidwell, K. K., and T. C. Osborn, 1992 Simple plant DNA isolation procedures, pp. 1-13 in Plant Genomes: Methods for Genetic and Physical Mapping, edited by J. S. Beckmann and T. C. Osborn. Springer Netherlands, Dordrecht.

Kim, D., B. Langmead, and S. L. Salzberg, 2015 HISAT: a fast spliced aligner with low memory requirements. Nat. Methods 12: 357-360.

Koch, L., 2016 Chicago HighRise for genome scaffolding. Nat. Rev. Genet. 17: 194.

Kranthi, K. R., 2018 Cotton production practices: snippets from global data 2017. The ICAC Recorder XXXVI: 4-14.

Krueger, F., 2015 Trim galore. A wrapper tool around Cutadapt and FastQC to consistently apply quality and adapter trimming to FastQ files.

Landolin, J. M., D. S. Johnson, N. D. Trinklein, S. F. Aldred, C. Medina et al., 2010 Sequence features that drive human promoter function and tissue specificity. Genome Res. 20: 890898.

Landt, S. G., G. K. Marinov, A. Kundaje, P. Kheradpour, F. Pauli et al., 2012 ChIP-seq guidelines and practices of the ENCODE and modENCODE consortia. Genome Res. 22: 1813-1831.

Langmead, B., and S. L. Salzberg, 2012 Fast gapped-read alignment with Bowtie 2. Nat. 
Methods 9: 357-359.

682

683

684

685

686

687

688

689

690

691

692

693

694

695

696

697

698

699

700

701

702

703

704

705

706

707

708

709

710

711

712

713

714

715

716

717

718

719

720

Lawrence, K., M. Olsen, T. Faske, R. Hutmacher, J. Muller et al., 2015 Cotton disease loss estimate committee report, 2014, pp. 188-190 in Proceedings of the 2015 Beltwide Cotton Conferences, San Antonio, TX. Cordova: National Cotton Council,.

Li, H., B. Handsaker, A. Wysoker, T. Fennell, J. Ruan et al., 2009 The Sequence Alignment/Map format and SAMtools. Bioinformatics 25: 2078-2079.

Lu, Z., B. T. Hofmeister, C. Vollmers, R. M. DuBois, and R. J. Schmitz, 2017 Combining ATAC-seq with nuclei sorting for discovery of cis-regulatory regions in plant genomes. Nucleic Acids Res. 45: e41.

Lu, Z., A. P. Marand, W. A. Ricci, C. L. Ethridge, X. Zhang et al., 2019 The prevalence, evolution and chromatin signatures of plant regulatory elements. Nat Plants.

Lyons, E., and M. Freeling, 2008 How to usefully compare homologous plant genes and chromosomes as DNA sequences: How to usefully compare plant genomes. Plant J. 53: 661-673.

Mapleson, D., L. Venturini, G. Kaithakottil, and D. Swarbreck, 2018 Efficient and accurate detection of splice junctions from RNA-seq with Portcullis. Gigascience 7.:

Marçais, G., A. L. Delcher, A. M. Phillippy, R. Coston, S. L. Salzberg et al., 2018 MUMmer4: A fast and versatile genome alignment system. PLoS Comput. Biol. 14: e1005944.

Martin, G. B., A. J. Bogdanove, and G. Sessa, 2003 Understanding the functions of plant disease resistance proteins. Annu. Rev. Plant Biol. 54: 23-61.

Nacoulima, N., J. P. Baudoin, and G. Mergeai, 2012 Introgression of improved fiber fineness trait in G. hirsutum L. from G. longicalyx Hutch. \& Lee. Commun. Agric. Appl. Biol. Sci. 77: 207-211.

Nichols, R. L., A. Bell, D. Stelly, N. Dighe, F. Robinson et al., 2010 Phenotypic and genetic evaluation of LONREN germplasm, pp. 798-799 in Proc. Beltwide Cotton Conf. New Orleans, LA,.

Novák, P., P. Neumann, and J. Macas, 2010 Graph-based clustering and characterization of repetitive sequences in next-generation sequencing data. BMC Bioinformatics 11: 378.

Ou, S., J. Chen, and N. Jiang, 2018 Assessing genome assembly quality using the LTR Assembly Index (LAI). Nucleic Acids Res. 46: e126.

Paterson, A. H., J. F. Wendel, H. Gundlach, H. Guo, J. Jenkins et al., 2012 Repeated polyploidization of Gossypium genomes and the evolution of spinnable cotton fibres. Nature 492: 423-427.

Pertea, M., G. M. Pertea, C. M. Antonescu, T.-C. Chang, J. T. Mendell et al., 2015 StringTie enables improved reconstruction of a transcriptome from RNA-seq reads. Nat. Biotechnol. 33: 290-295.

Phillips, L. L., 1966 the cytology and phylogenetics of the diploid species of GOSSYPIUM. Am. J. Bot. 53: 328-335.

Putnam, N. H., B. L. O’Connell, J. C. Stites, B. J. Rice, M. Blanchette et al., 2016 Chromosomescale shotgun assembly using an in vitro method for long-range linkage. Genome Res. 26: 
342-350.

Quinlan, A. R., 2014 BEDTools: The Swiss-Army Tool for Genome Feature Analysis. Curr. Protoc. Bioinformatics 47: 11.12.1-34. generation web server for deep-sequencing data analysis. Nucleic Acids Res. 44: W160-5. Statistical Computing., Vienna, Austria.

R Development Core Team, R., and Others, 2011 R: A language and environment for statistical computing.

Ricci, W. A., Z. Lu, L. Ji, A. P. Marand, C. L. Ethridge et al., 2019 Widespread long-range cisregulatory elements in the maize genome. Nat Plants 5: 1237-1249.

Robinson, A. F., 2007 Reniform in U.S. cotton: when, where, why, and some remedies. Annu. Rev. Phytopathol. 45: 263-288.

Robinson, A. F., R. N. Inserra, E. P. Caswell-Chen, N. Vovlas, and A. Troccoli, 1997 Rotylenchulus Species: Identification, Distribution, Host Ranges, and Crop Plant Resistance | Nematropica. Nematropica 27: 127-180. resistance gene $\mathrm{Mi}$ of tomato confers resistance against the potato aphid. Proc. Natl. Acad. Sci. U. S. A. 95: 9750-9754.

Schliep, K. P., 2011 phangorn: phylogenetic analysis in R. Bioinformatics 27: 592-593.

Smit, A. F. A., R. Hubley, and P. Green, 2015 RepeatMasker Open-4.0. 2013--2015.

Sohani, M. M., P. M. Schenk, C. J. Schultz, and O. Schmidt, 2009 Phylogenetic and transcriptional analysis of a strictosidine synthase-like gene family in Arabidopsis thaliana reveals involvement in plant defence responses. Plant Biol. 11: 105-117.

Stanke, M., O. Keller, I. Gunduz, A. Hayes, S. Waack et al., 2006 AUGUSTUS: ab initio prediction of alternative transcripts. Nucleic Acids Res. 34: W435-9.

The Gene Ontology Consortium, 2019 The Gene Ontology Resource: 20 years and still GOing strong. Nucleic Acids Res. 47: D330-D338.

Udall, J. A., E. Long, C. Hanson, D. Yuan, T. Ramaraj et al., 2019 De Novo Genome Sequence Assemblies of Gossypium raimondii and Gossypium turneri. G3 g3.400392.2019.

UniProt Consortium, 2008 The universal protein resource (UniProt). Nucleic Acids Res. 36: D190-5.

Venturini, L., S. Caim, G. G. Kaithakottil, D. L. Mapleson, and D. Swarbreck, 2018 Leveraging multiple transcriptome assembly methods for improved gene structure annotation. Gigascience 7.:

Wang, J., J. Zhuang, S. Iyer, X. Lin, T. W. Whitfield et al., 2012 Sequence features and chromatin structure around the genomic regions bound by 119 human transcription factors. Genome Research 22: 1798-1812. "Reference Genome Sequences of Two Cultivated Allotetraploid Cottons, Gossypium 
hirsutum and Gossypium barbadense.” Nature Genetics 51 (2): 224-29.

Waterhouse, R. M., M. Seppey, F. A. Simão, M. Manni, P. Ioannidis et al., 2017 BUSCO

763 applications from quality assessments to gene prediction and phylogenomics. Mol. Biol. Evol.

Wendel, J. F., and V. A. Albert, 1992 Phylogenetics of the Cotton Genus (Gossypium): Character-State Weighted Parsimony Analysis of Chloroplast-DNA Restriction Site Data and Its Systematic and Biogeographic Implications. Syst. Bot. 17: 115-143.

Wendel, J. F., and C. E. Grover, 2015 Taxonomy and Evolution of the Cotton Genus, Gossypium, pp. 25-44 in Cotton, Agronomy Monograph, American Society of Agronomy, Inc., Crop Science Society of America, Inc., and Soil Science Society of America, Inc., Madison, WI.

Wickham, H., 2016 ggplot2: Elegant Graphics for Data Analysis. Springer-Verlag New York.

Wickham, H., R. Francois, L. Henry, K. Müller, and Others, 2015 dplyr: A grammar of data manipulation. $\mathrm{R}$ package version 0.4 3.:

Wu, T. D., and C. K. Watanabe, 2005 GMAP: a genomic mapping and alignment program for mRNA and EST sequences. Bioinformatics 21: 1859-1875.

Yandell, M., and D. Ence, 2012 A beginner's guide to eukaryotic genome annotation. Nat. Rev. Genet. 13: 329-342.

Yik, C. P., and W. Birchfield, 1984 Resistant Germplasm in Gossypium Species and Related Plants to Rotylenchulus reniformis. J. Nematol. 16: 146-153.

Yu, J., S. Jung, C.-H. Cheng, S. P. Ficklin, T. Lee et al., 2014 CottonGen: a genomics, genetics and breeding database for cotton research. Nucleic Acids Res. 42: D1229-36.

Yu, G., L.-G. Wang, and Q.-Y. He, 2015 ChIPseeker: an R/Bioconductor package for ChIP peak annotation, comparison and visualization. Bioinformatics 31: 2382-2383.

Zhang, Y., T. Liu, C. A. Meyer, J. Eeckhoute, D. S. Johnson et al., 2008 Model-based analysis of ChIP-Seq (MACS). Genome Biol. 9: R137.

Zheng, X., K. A. Hoegenauer, J. Quintana, A. A. Bell, A. M. Hulse-Kemp et al., 2016 SNPBased MAS in Cotton under Depressed-Recombination for RenLon_Flanking Recombinants: Results and Inferences on Wide-Cross Breeding Strategies. Crop Sci. 56: 1526-1539. 